\title{
The Impact of Chemotherapy on the EORTC QLQ-C30 and LC-13 Quality of Life Scales in Patients with Lung Cancer
}

Özgün Araştırma Research Article

Received/Geliş: 31.01 .2020 Accepted/Kabul: 27.09.2020 First Online: 28.09 .2021

Ahmet Emin Erbaycu izmir Bakırçay Üniversitesi Tıp Fakültesi, Göğüs Hastalıkları Anabilim Dalı, izmir, Turkey afumetsu67@gmail.com ORCID: 0000-0001-6618-6774

M. Güvençli 0000-0002-1864-7326 E. Yalnız 0000-0002-3231-9513

B. Kömürcüoğlu 0000-0002-2877-242X

G. Karakurt 0000-0001-6490-8139 SBÜ. İzmir Suat Seren Göğüs Hastalıkları ve Cerrahisi Eğitim ve Araștırma Hastanesi, Göğüs Hastalıkları Anabilim Dalı, izmir, Türkiye

*Ulusal Akciğer Sağlığı Kongresi 2016 Kongresi'nde Sözlü Sunum olarak sunulmuştur.

Cite as: Güvençli $M$, Yalnız $E$, Kömürcüoğlu Cite as: Güvençli $M$, Yalnız $E$, Komürcüoğ $B$, Erbaycu AE, Karakurt G. The impact of chemotherapy on the EORTC QLQ-C3O and LC-13 quality of life scales in patients with lung cancer. Tepecik Eğit. ve Araşt. Hast. Dergis 2021;31(3):344-54.

\section{Akciğer Kanserli Hastalarda Kemoterapinin EORTC QLQ-C30 ve LC-13 Yaşam Kalitesi Ölçeklerine Etkisi}

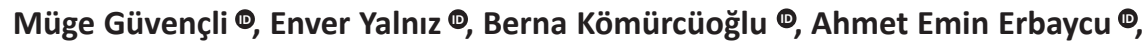 \\ Gamze Karakurt (1)
}

\begin{abstract}
Objective: The concept of quality of life (QoL) in lung cancer includes many physical, psychological and social components. We aimed to assess the effect of chemotherapy (CT) on QoL of lung cancer patients using QoL scales.

Methods: Fifty inoperable lung cancer patients who were newly diagnosed and taken into a CT plan were included. Patients were followed in terms of responsiveness and toxicity. Turkish versions of the EORTC QLQ-C3O and LC13 scales were used before every cycles.

Results: The average age was 60.1 years. There was no difference between QoL and age/income levels. The assessment of physical, social and occupational functions and overall health status of the male patients was better than female. Overall health status without comorbidity was better in the first cycle CT. Chemotherapy led to deterioration in social functions and economic status together with increase in neuropathy, constipation and hair loss. Patients with complete or partial response to treatment were observed to have better physical, occupational, emotional, cognitive and social functions, economic status and overall health; less fatigue, pain, shortness of breath, neuropathy and better appetite. Toxicities were found to affect the QLQ C3O and LC13 scales adversely. Conclusion: Presence of comorbidity, low education levels, socioeconomic status and CT induced hematologic/ gastrointestinal toxicities are the major parameters affect QOL in lung cancer. Chemotherapy leads to deterioration in social functions, increase in adverse events as well as worsening in economic status. Radiologic complete or partial response and small cell carcinoma are states in which parameters of QoL are affected positively by chemotherapy.
\end{abstract}

Keywords: Lung cancer, chemotherapy, EORTC QLQ-C30, LC13, quality of life

öz

Amaç: Akciğer kanseri hastalarında yaşam kalitesi kavramı; fiziksel, psikolojik ve sosyal olmak üzere pek çok komponenti içermektedir. Bu çalışmada akciğer kanserli hastalarda kemoterapinin yaşam kalitesi üzerine etkisinin yaşam kalitesi ölçekleri kullanılarak belirlenmesi amaçlanmıştır.

Yöntem: Araştırmaya yeni tanı alan ve kemoterapi planlanan 50 inoperabl akciğer kanserli hasta alındı. Hastalar tedaviye yanıt ve toksisite yönünden izleme alındı. EORTC QLQ-C30 ve LC13 ölçeklerinin Türkçe sürümleri her siklus öncesi uygulandı.

Bulgular: Yaş ortalaması 60.1 yıldı. Yaș ve gelir düzeyi ile yaşam kalitesi parametreleri arasında fark bulunmadı. Erkeklerin; fiziksel, sosyal ve uğraş fonksiyonlarının ve genel sağlık durumu değerlendirmesi kadınlara göre daha iyi idi. Komorbiditesi olmayanların, 1. Siklus KT'de genel sağlık durumu değerlendirmesi daha iyi idi. Kemoterapi ile sosyal fonksiyonlarda, ekonomik durumda kötüleşme, nöropati, kabızlık ve saç dökülmesinde artış saptandı. Tedaviye tam veya kısmi yanıt elde edilen hastaların, fiziksel, uğraş, duygusal, kavrama, sosyal fonksiyonlarının, ekonomik durumlarının ve genel durum değerlendirmesinin daha iyi olduğu, yorgunluk, ağrı, nefes darlığı, nöropatinin daha az olduğu ve iştahın daha iyi olduğu belirlendi. Toksisitelerin QLQ C30 ve LC13 ölçeklerini olumsuz yönde etkilediği görüldü.

Sonuç: Akciğer kanserinde komorbidite varlığı, düşük eğitim düzeyi, sosyoekonomik durum ve kemoterapiye bağlı hematolojik/gastrointestinal toksisiteler yașam kalitesini etkileyen en önemli parametrelerdir. Kemoterapi ile sosyal fonksiyonlar ve ekonomik parametrelerde kötüleşme ile birlikte yan etkilerde artış izlenmiştir. Radyolojik tam veya kısmi yanıt, küçük hücreli karsinom hücre tipi kemoterapi ile yaşam kalitesi parametrelerinin olumlu etkilendiği durumlardır.

Anahtar kelimeler: Akciğer kanseri, kemoterapi, EORTC QLQ-C30, LC13, yaşam kalitesi

(c) Telif hakkı T.C. Sağık Bakanlığı İzmir Tepecik Eğit. ve Araşt. Hastanesi. Logos Tıp Yayıncılık tarafindan yayınlanmaktadır. Bu dergide yayınlanan bütün makaleler Creative Commons Attf-GayriTicari 4.0 Uluslararası Lisansı ile lisanslanmıştr.

(c) Copyright Association of Publication of the T.C. Ministry of Health izmir Tepecik Education and Research Hospital. This journal published by Logos Medical Publishing.

Licenced by Creative Commons Attribution-NonCommercial 4.0 International (CC BY) 


\section{INTRODUCTION}

Integrating quality of life (QoL) assessments into the clinical routine in lung cancer patients may provide a subjective measure of symptom severity. It also facilitate patients and their families to participate in medical decisions ${ }^{(1)}$. Both disease specific and generic instruments are available for QoL of lung cancer patients. The comparison of QoL scores between lung cancer patients and the healthy population may be done by using generic instruments (2). Recently, QoL becomes a more important outcome measure in assessment of the best standard of care for patients. QoL may be evaluated as a primary endpoint of treatment both in clinical practice and in clinical trials to define meaningful response ${ }^{(3)}$.

Due to serious side effect profiles of treatment models such as chemotherapy (CT) and radiotherapy, serious treatment induced side effects come up and the QoL deteriorates. Besides the success of cancer treatment, response rates and survival time; use of QoL scales has become a significant criterion for doctors in the determination of treatment method and evaluation of the response by measuring the patient's physical, emotional and social functions ${ }^{(4-6)}$.

In patients with advanced lung cancer, clinicians should enhance their ability to notice patients' elevated risk of poor QoL during CT. They should also detect and manage the related physical symptoms and side effects, strengthen patients' social support and control the anxiety and depressive symptoms (7).

The present study aims to to evaluate the effect of the CT on the QoL of lung cancer patients by using the lung cancer specific QoL module.

\section{MATERIAL and METHOD}

The study was a prospective, descriptive clinical study and was carried out between 01/08/2014 and 01/06/2015.

\section{Study Population and Sample}

The study sample consisted of inoperable (stage IIIAIIIB-IV) patients who were radiologically and histopathologically diagnosed with lung cancer (small cell and non-small cell carcinoma) (SCLC, NSCLC) and given $\mathrm{CT}$. The fact that the patients had received or would receive palliative treatment was not an obstacle for the study. Patients who were suggested surgical treatment, those followed without treatment, and patients failing to fill in questionnaire forms / having communicational problems were excluded. Patients; accepted to participate, to come for checkups, were literate and able to read/fill in test forms were included.

In order to conduct the study, permission was taken from the Local Ethics Committee and written informed consent forms were completed by all patients accepted to participate.

\section{Data Collection}

Before treatment, demographic data, comorbidity, smoking and alcohol use, performances observed by the doctor, histologically/histopathologically confirmed tumor cell type and the stage of disease were collected. Turkish versions of the EORTC QLQ C30 and LC13 scales were given before the treatment, with face-to-face interviewing technique. Each interview lasted approximately 30 minutes ${ }^{(8)}$.

$7^{\text {th }}$ edition tumor-node-metastasis (TNM) staging system were used ${ }^{(7)}$. Response and ECOG/Karnofsky performance scores assessment was performed after $2^{\text {nd }}-4^{\text {th }}$ cycles. The EORTC QLQ-C30 and LC13 scales were applied prior to each cycle with face to face in a vacant and quiet room before examination. Performance was recorded by using ECOG and Karnofsky ${ }^{(9,10)}$. During four cycle (per three weeks) $\mathrm{CT}$, we used The Response Evaluation Criteria in Solid Tumors (RECIST) and the Common Terminology Criteria for Adverse Events v3.0 (CTCAE) (five categories based on the degree) ${ }^{(11)}$. Chemotherapy induced adverse effects were recorded following each cycle.

The EORTC-QLQ-30 Scale (European Organization for 


\section{Research and Treatment of Cancer QoL Questionnaire)}

The questionnaire has 30 questions ( $Q$ ) and incorporates functional and symptom scales. The functional scale includes six subscales; physical (Q1-5), role $(Q 6,7)$, cognitive $(Q 20,25)$, emotional (Q21-24), social $(Q 26,27)$, global QOL $(Q 29,30)$. Symptom scale consists of symptoms of fatigue $(Q 10,12,18)$, nausea and vomiting $(\mathrm{Q} 14,15)$, pain $(\mathrm{Q} 9,19)$, dyspnea $(\mathrm{Q} 8)$, insomnia (Q11), loss of appetite (Q13), constipation (Q16), diarrhea (Q17) and perceived financial impact of the disease (Q28). Of the 30 items on the scale, 28 are designed as a 4-point Likert type scale and scored as Not at All: 1, A Little: 2, Quite a Bit: 3, Very Much: 4 points. Q29 asks the patients to rate their health status on a scale rated from 1 to 7 (1: very bad and 7: excellent) and Q30 wants them to assess their global QoL. Q29.30 make up the overall well-being section of the scale, and high scores obtained over this section indicate high QoL while low scores show that QoL decreases. In the functional area and symptoms section, on the other hand, low scores reflect high QoL whereas high scores are indicators of low QoL ${ }^{(12)}$. The scale was confirmed for validity and reliability for the Turkish society ${ }^{(13)}$.

\section{The EORTC QLQ-LC13 Scale}

It measures treatment symptoms and consists of 13 items. The questions assess dyspnea $(Q 3,4,5)$, coughing (Q1), haemoptysis (Q2), sore mouth (Q6), dysphagia (Q7), peripheral neuropathy (Q8), alopecia (Q9), chest pain (Q10), pain in arms and shoulders (Q11), other types of pain (Q12) and any medication taken for pain (Q13) ${ }^{(14)}$.

\section{Data Analysis and Assessment Techniques}

The data obtained from the study were analyzed using SPSS 18 (Statistical Package For Social Sciences). Mean, standard deviation, median, minimum and maximum values pertaining to the continuous variables were presented. These variables and their subgroups were analyzed for normal distribution. Considering the graphical research and normality tests and the sample size, it was concluded that not all the variables met the conditions required for nor- mal distribution. Thus, non-parametric test methods were employed for the comparisons of these variables.

Independent groups were compared using Mann Whitney test; while the comparisons of the variables obtained from repeated measures were carried out employing Friedman and Wilcoxon tests. The relations between variables were analyzed with the nonparametric correlation method.

Frequency tables were created for class variables and the equality of the distribution between groups was tested using chi-square tests. Class variables were measured repeatedly and the change was studied with Mc-Nemar test method. In all statistical comparison tests, margin of type 1 error was accepted as $\alpha: 0,05$ and was tested as two-way.

\section{RESULTS}

General characteristics are presented in Table 1.60\% had equal income and expenses, 34\% had lower income than their expenses and $6 \%$ had higher income than their expenses. No difference was observed in the ECOG and KPS at diagnosis, following two cycles and at the end of the treatment ( $p>0.05)$.

$44(88 \%)$ of the patients had completed four cycles of treatment, one $(2 \%)$ died after the $1^{\text {st }}$ cycle, two (4\%) died after the $2^{\text {nd }}$. Due to low performance, CT was not given to two (4\%) after the $2^{\text {nd }}$ cycle and to one $(2 \%)$ after the $3^{\text {rd }}$ cycle. Response assessments following two cycles of CT showed partial response in $15(30 \%)$, progression in $16(32 \%)$, stable response in $17(34 \%)$ and complete response in one (2\%). In the response assessments following four cycles, six failed to complete four cycles due to varying reasons. Fifteen (30\%) had partial response, eight (16\%) had progression, $19(38 \%)$ had stable response and two (4\%) had complete response.

No correlation was found between age and QoL $(p>0.05)$. Men had better physical and occupational 
functions in the $1^{\text {st }}$ and $2^{\text {nd }}$ cycle $\mathrm{CT}$, and social functions and global health status of men were better than women in the $1^{\text {st }}$ cycle $(p<0.05)$. No difference was found in other parameters. Patients completed primary and further education had better social functions in the $1^{\text {st }}$ cycle $(p=0.024)$, better physical

Table 1. General characteristics of the patients with lung cancer.

\begin{tabular}{|c|c|c|}
\hline Feature & $\mathbf{N}$ & $\%$ \\
\hline Age, years & $60.1 \pm 8,1$ & \\
\hline Cigarette smoking, package years & $41 \pm 24$ & \\
\hline \multicolumn{3}{|l|}{ Gender } \\
\hline Men & 40 & 80 \\
\hline Women & 10 & 20 \\
\hline \multicolumn{3}{|l|}{ Education } \\
\hline Primary & 39 & 78 \\
\hline Secondary & 7 & 14 \\
\hline High school & 2 & 4 \\
\hline College or higher levels & 2 & 4 \\
\hline \multicolumn{3}{|l|}{ Histopathology } \\
\hline Small cell & 14 & 28 \\
\hline Squamous cell & 4 & 8 \\
\hline Adeno carcinoma & 10 & 20 \\
\hline $\begin{array}{l}\text { Non-small cell (subtype not } \\
\text { identified) }\end{array}$ & 22 & 44 \\
\hline \multicolumn{3}{|l|}{ Performance Status } \\
\hline ECOG 0 & 10 & 20 \\
\hline ECOG I & 23 & 46 \\
\hline ECOG II & 14 & 28 \\
\hline ECOG III & 3 & 6 \\
\hline Karnofsky, $>80 \%$ & 40 & 80 \\
\hline \multicolumn{3}{|l|}{ Comorbidity $^{\dagger}$} \\
\hline Yes & 34 & 68 \\
\hline No & 16 & 32 \\
\hline \multicolumn{3}{|l|}{ Stage at Diagnosis } \\
\hline Stage $3 A$ & 8 & 16 \\
\hline Stage $3 B$ & 8 & 16 \\
\hline Stage 4 & 20 & 40 \\
\hline Metastatic * & 10 & 20 \\
\hline Limited * & 4 & 8 \\
\hline Total & 50 & \\
\hline
\end{tabular}

*Small cell lung carcinoma

+Diabetes mellitus, hypertension, chronic heart disease, chronic obstructive lung disease functions in the $3^{\text {rd }}$ cycle $(p=0.040)$ and better occupational functions in the $3^{\text {rd }}(p=0.022)$ and $4^{\text {th }}$ cycles $(p=0.023)$. No difference was found for other scores according to number of cycles in emotional function, cognitive function and global health status evaluations $(p>0.05)$.

QoL did not vary by income status ( $p>0.05$ ). Global health status evaluation with no comorbidity was better in the $1^{\text {st }}$ cycle in comparison with those having comorbidities $(p<0.05)$, whereas no significant difference was found in the other functions ( $p>0.05)$.

According to QLQ-C30 scale; social functions deteriorated $(p=0.000)$ while constipation $(p=0.000)$ and financial distress $(p=0.000)$ increased with $C T$, and LC13 scale showed that hair loss $(p=0.000)$ and neuropathy $(p=0.000)$ increased together with CT. Other parameters of both scales did not differ $(p>0.05)$.

In the cell type-based analysis, occupational function in the $1^{\text {st }}$ cycle was better in NSCLC than SCLC $(p=0.028)$. In SCLC, physical functions $(p=0.030$ and $p=0.015)$, occupational functions $(p=0.042$ and $p=0.023)$ and global health status $(p=0,018$ and $p=0.006$ ) evaluation were better in the $3^{\text {rd }}$ and $4^{\text {th }}$ cycles; and that fatigue ( $p=0.025$ and $p=0.005)$, pain $(p=0.020$ and $p=0.028)$ and loss of appetite $(p=0.041$ and $p=0.079$ ) symptoms were better in the $3^{\text {rd }}$ and $4^{\text {th }}$ cycles. Other parameters of both scales did not differ $(p>0.05)$. With the LC13 scale, neuropathy $(p=0.000)$ was experienced more severely in the $3^{\text {rd }}$ and $4^{\text {th }}$ cycles, alopecia $(p=0.042)$ in the $1^{\text {st }}$ cycle and pain in arms and shoulders $(p=0.028)$ in the $3^{\text {rd }}$ cycle in NSCLC. Other parameters were similar between tumor cell types ( $p>0.05)$.

In complete/partial response group, physical functions in cycles 2,3,4; occupational functions in cycles 3 and 4; emotional functions in cycle 3 , cognitive functions in cycles 3 and 4 , financial status and social functions in cycle 1; and global status evaluations were better than stable/progressive. Complete/partial group had less pain in cycle 4, experienced less 
Table 2. QLQ-C30 and toxicity correlation in the $2^{\text {nd }}$ cycle.

\begin{tabular}{|c|c|c|c|c|c|c|c|c|}
\hline & & Anemia & Leucopenia & Neutropenia & $\begin{array}{l}\text { Thromboc } \\
\text { ytopenia }\end{array}$ & $\begin{array}{l}\text { Weight } \\
\text { loss }\end{array}$ & Nausea & Vomiting \\
\hline \multirow{3}{*}{$\begin{array}{l}\text { Physical } \\
\text { Function } \\
\text { (Total) }\end{array}$} & CorrelationCoefficient & 0,217 & 0,240 & 0,197 & ,327(*) &, $535(* *)$ &, $422(* *)$ &, $431(* *)$ \\
\hline & Sig. (2-tailed) & 0,134 & 0,097 & 0,176 & 0,022 & 0,000 & 0,003 & 0,002 \\
\hline & $\mathrm{N}$ & 49 & 49 & 49 & 49 & 49 & 49 & 49 \\
\hline \multirow{3}{*}{$\begin{array}{l}\text { Occupational } \\
\text { Function } \\
\text { (Total) }\end{array}$} & CorrelationCoefficient & 0,231 & 0,176 & 0,244 & ,337(*) &, $379(* *)$ & 0,261 & ,341(*) \\
\hline & Sig. (2-tailed) & 0,110 & 0,227 & 0,092 & 0,018 & 0,007 & 0,070 & 0,017 \\
\hline & $\mathrm{N}$ & 49 & 49 & 49 & 49 & 49 & 49 & 49 \\
\hline \multirow{3}{*}{$\begin{array}{l}\text { Emotional } \\
\text { Function } \\
\text { (Total) }\end{array}$} & CorrelationCoefficient &, $303(*)$ & 0,205 & 0,155 & 0,279 &, $392(* *)$ &, $323(*)$ &, $385(* *)$ \\
\hline & Sig. (2-tailed) & 0,034 & 0,158 & 0,289 & 0,052 & 0,005 & 0,024 & 0,006 \\
\hline & $\mathrm{N}$ & 49 & 49 & 49 & 49 & 49 & 49 & 49 \\
\hline \multirow{3}{*}{$\begin{array}{l}\text { Cognitive } \\
\text { Function } \\
\text { (Total) }\end{array}$} & CorrelationCoefficient & 0,047 & 0,199 & 0,204 & $-0,074$ & 0,233 &, $287(*)$ & 0,182 \\
\hline & Sig. (2-tailed) & 0,749 & 0,171 & 0,161 & 0,612 & 0,106 & 0,045 & 0,211 \\
\hline & $\mathrm{N}$ & 49 & 49 & 49 & 49 & 49 & 49 & 49 \\
\hline \multirow{3}{*}{$\begin{array}{l}\text { Social } \\
\text { Function } \\
\text { (Total) }\end{array}$} & CorrelationCoefficient & 0,277 & 0,205 & 0,204 & 0,242 &, $482(* *)$ & 0,248 &, $327(*)$ \\
\hline & Sig. (2-tailed) & 0,054 & 0,158 & 0,160 & 0,094 & 0,000 & 0,085 & 0,022 \\
\hline & $\mathrm{N}$ & 49 & 49 & 49 & 49 & 49 & 49 & 49 \\
\hline \multirow{3}{*}{$\begin{array}{l}\text { Fatigue } \\
\text { (Total) }\end{array}$} & CorrelationCoefficient & 0,185 & ,286(*) & ,298(*) & ,301(*) &, $556(* *)$ &, $498(* *)$ &, $431(* *)$ \\
\hline & Sig. (2-tailed) & 0,203 & 0,046 & 0,038 & 0,036 & 0,000 & 0,000 & 0,002 \\
\hline & $\mathrm{N}$ & 49 & 49 & 49 & 49 & 49 & 49 & 49 \\
\hline \multirow[t]{3}{*}{ Pain (Total) } & CorrelationCoefficient & 0,088 & 0,181 & 0,255 & ,292(*) & ,308(*) &, $408(* *)$ &, $559(* *)$ \\
\hline & Sig. (2-tailed) & 0,550 & 0,214 & 0,077 & 0,042 & 0,031 & 0,004 & 0,000 \\
\hline & $\mathrm{N}$ & 49 & 49 & 49 & 49 & 49 & 49 & 49 \\
\hline \multirow{3}{*}{$\begin{array}{l}\text { Nausea/Vomi } \\
\text { ting (Total) }\end{array}$} & CorrelationCoefficient & ,291(*) & ,311(*) &, $298\left(^{*}\right)$ & 0,260 &, $583(* *)$ &, $872(* *)$ &, $719(* *)$ \\
\hline & Sig. (2-tailed) & 0,043 & 0,030 & 0,038 & 0,071 & 0,000 & 0,000 & 0,000 \\
\hline & $\mathrm{N}$ & 49 & 49 & 49 & 49 & 49 & 49 & 49 \\
\hline \multirow[t]{3}{*}{ Dyspnea } & CorrelationCoefficient & 0,132 & 0,158 & 0,229 & 0,234 &, $312(*)$ &, $386(* *)$ &, $381(* *)$ \\
\hline & Sig. (2-tailed) & 0,368 & 0,278 & 0,113 & 0,105 & 0,029 & 0,006 & 0,007 \\
\hline & $\mathrm{N}$ & 49 & 49 & 49 & 49 & 49 & 49 & 49 \\
\hline \multirow[t]{3}{*}{ Insomnia } & CorrelationCoefficient & 0,054 & 0,120 & 0,124 & 0,222 &, $521(* *)$ &, $436(* *)$ &, $325(*)$ \\
\hline & Sig. (2-tailed) & 0,714 & 0,410 & 0,394 & 0,125 & 0,000 & 0,002 & 0,023 \\
\hline & $\mathrm{N}$ & 49 & 49 & 49 & 49 & 49 & 49 & 49 \\
\hline \multirow{3}{*}{$\begin{array}{ll}\text { Loss } \\
\text { appetite }\end{array}$} & CorrelationCoefficient & 0,226 & 0,159 & 0,135 & 0,161 &, $496(* *)$ &, $495(* *)$ &, $439(* *)$ \\
\hline & Sig. (2-tailed) & 0,118 & 0,275 & 0,354 & 0,270 & 0,000 & 0,000 & 0,002 \\
\hline & $\mathrm{N}$ & 49 & 49 & 49 & 49 & 49 & 49 & 49 \\
\hline \multirow[t]{3}{*}{ Constipation } & CorrelationCoefficient & $-0,026$ & 0,199 & 0,127 & 0,051 & 0,101 & 0,106 & 0,099 \\
\hline & Sig. (2-tailed) & 0,862 & 0,169 & 0,383 & 0,726 & 0,491 & 0,470 & 0,501 \\
\hline & $\mathrm{N}$ & 49 & 49 & 49 & 49 & 49 & 49 & 49 \\
\hline \multirow[t]{3}{*}{ Diarrhea } & CorrelationCoefficient & 0,056 & $-0,083$ & $-0,205$ & $-0,188$ & 0,180 & $-0,052$ & $-0,101$ \\
\hline & Sig. (2-tailed) & 0,702 & 0,570 & 0,157 & 0,196 & 0,217 & 0,722 & 0,491 \\
\hline & $\mathrm{N}$ & 49 & 49 & 49 & 49 & 49 & 49 & 49 \\
\hline \multirow{3}{*}{$\begin{array}{l}\text { Global } \\
\text { health status } \\
\text { evaluation }\end{array}$} & CorrelationCoefficient & $-0,214$ & $-0,148$ & $-0,142$ & $-0,117$ &,$- 383(* *)$ &,$- 385(* *)$ &,$- 380(* *)$ \\
\hline & Sig. (2-tailed) & 0,141 & 0,309 & 0,332 & 0,425 & 0,007 & 0,006 & 0,007 \\
\hline & $\mathrm{N}$ & 49 & 49 & 49 & 49 & 49 & 49 & 49 \\
\hline
\end{tabular}

${ }^{*} p<0.05,{ }^{*} p<0.01$ 
Table 3. LC13 and toxicity correlation in the $2^{\text {nd }}$ cycle.

\begin{tabular}{|c|c|c|c|c|c|c|c|c|}
\hline & & Anemia & Leucopenia & Neutropenia & Thrombocytopenia & $\begin{array}{l}\text { Weight } \\
\text { loss }\end{array}$ & Nausea & Vomiting \\
\hline \multirow[t]{3}{*}{ Coughing 2} & CorrelationCoefficient & $-0,003$ & 0,000 & $-0,058$ & $-0,062$ &, $453(* *)$ &, $371(* *)$ & 0,264 \\
\hline & Sig. (2-tailed) & 0,982 & 0,998 & 0,692 & 0,671 & 0,001 & 0,009 & 0,067 \\
\hline & $\mathrm{N}$ & 49 & 49 & 49 & 49 & 49 & 49 & 49 \\
\hline \multirow[t]{3}{*}{ Haemoptysis 2} & CorrelationCoefficient & 0,105 & ,310(*) &, $364(*)$ &, $287(*)$ &, $501(* *)$ & ,463(**) &, $421(* *)$ \\
\hline & Sig. (2-tailed) & 0,471 & 0,030 & 0,010 & 0,045 & 0,000 & 0,001 & 0,003 \\
\hline & $\mathrm{N}$ & 49 & 49 & 49 & 49 & 49 & 49 & 49 \\
\hline \multirow[t]{3}{*}{ Dyspnea (ort) 2} & CorrelationCoefficient & 0,212 & 0,225 & 0,231 &, $300(*)$ &, $546(* *)$ &, $552(* *)$ &, $446(* *)$ \\
\hline & Sig. (2-tailed) & 0,144 & 0,121 & 0,110 & 0,037 & 0,000 & 0,000 & 0,001 \\
\hline & $\mathrm{N}$ & 49 & 49 & 49 & 49 & 49 & 49 & 49 \\
\hline \multirow[t]{3}{*}{ Sore mouth 2} & CorrelationCoefficient & $-0,064$ & 0,271 &, $321(*)$ & 0,243 & ,302(*) &, $287(*)$ &, $295(*)$ \\
\hline & Sig. (2-tailed) & 0,664 & 0,060 & 0,025 & 0,093 & 0,035 & 0,046 & 0,039 \\
\hline & $\mathrm{N}$ & 49 & 49 & 49 & 49 & 49 & 49 & 49 \\
\hline \multirow[t]{3}{*}{ Dysphagia2 } & CorrelationCoefficient & $-0,038$ & 0,197 & 0,233 & 0,259 & ,298(*) &, $451(* *)$ &, $549(* *)$ \\
\hline & Sig. (2-tailed) & 0,797 & 0,176 & 0,107 & 0,073 & 0,038 & 0,001 & 0,000 \\
\hline & $\mathrm{N}$ & 49 & 49 & 49 & 49 & 49 & 49 & 49 \\
\hline \multirow[t]{3}{*}{ Neuropathy 2} & CorrelationCoefficient & 0,038 &, $285(*)$ & 0,279 & 0,248 &, $367(* *)$ &, $350(*)$ & 0,231 \\
\hline & Sig. (2-tailed) & 0,793 & 0,047 & 0,052 & 0,086 & 0,010 & 0,014 & 0,111 \\
\hline & $\mathrm{N}$ & 49 & 49 & 49 & 49 & 49 & 49 & 49 \\
\hline \multirow[t]{3}{*}{ Alopecia 2} & CorrelationCoefficient & $-0,077$ & $-0,115$ & $-0,102$ & 0,137 & 0,113 & $-0,071$ & $-0,129$ \\
\hline & Sig. (2-tailed) & 0,598 & 0,432 & 0,487 & 0,348 & 0,438 & 0,629 & 0,377 \\
\hline & $\mathrm{N}$ & 49 & 49 & 49 & 49 & 49 & 49 & 49 \\
\hline \multirow[t]{3}{*}{ Chest pain 2} & CorrelationCoefficient & 0,157 & 0,227 & ,289(*) & 0,237 &, $282(*)$ & 0,279 & ,328(*) \\
\hline & Sig. (2-tailed) & 0,280 & 0,117 & 0,044 & 0,102 & 0,050 & 0,052 & 0,021 \\
\hline & $\mathrm{N}$ & 49 & 49 & 49 & 49 & 49 & 49 & 49 \\
\hline \multirow{3}{*}{$\begin{array}{l}\text { Pain in arms and } \\
\text { shoulders } 2\end{array}$} & CorrelationCoefficient & 0,012 & 0,073 & 0,127 & 0,122 & 0,264 &, $283(*)$ & ,329(*) \\
\hline & Sig. (2-tailed) & 0,933 & 0,620 & 0,383 & 0,402 & 0,066 & 0,049 & 0,021 \\
\hline & $\mathrm{N}$ & 49 & 49 & 49 & 49 & 49 & 49 & 49 \\
\hline
\end{tabular}

${ }^{*} p<0.05,{ }^{*} p<0.01$

dyspnea and fatigue in cycles 3 and 4 , had better appetite in cycles 3 and 4 , less neuropathy in cycles 2,3 and 4 and less pain in arms and shoulders in cycle $3(p<0.05)$.

In $2^{\text {nd }}$ cycle, strong positive correlations were found between deterioration in physical functions and weight loss, nausea and vomiting; between deterioration in occupational functions and weight loss; between deterioration in emotional functions and weight loss/vomiting; and between deterioration in social functions and weight loss. Strong positive correlations were found between fatigue and nausea, vomiting and weight loss; between insomnia and 
Table 4. QLQ-C30 and toxicity correlation in the $4^{\text {th }}$ cycle.

\begin{tabular}{|c|c|c|c|c|c|c|c|c|}
\hline & & $\begin{array}{l}\text { Anemi } \\
\text { a }\end{array}$ & $\begin{array}{l}\text { Leucopeni } \\
\text { a }\end{array}$ & $\begin{array}{l}\text { Neutropeni } \\
\text { a }\end{array}$ & $\begin{array}{l}\text { Thrombocy } \\
\text { topenia }\end{array}$ & Weight loss & Nausea & Vomiting \\
\hline \multirow{3}{*}{$\begin{array}{l}\text { Physical } \\
\text { Function } \\
\text { (Total) } 4\end{array}$} & CorrelationCoefficient & 0,113 & $-0,125$ & $-0,125$ & 0,107 &, $428(* *)$ &, $432(* *)$ &, $410(* *)$ \\
\hline & Sig. (2-tailed) & 0,465 & 0,417 & 0,417 & 0,489 & 0,004 & 0,003 & 0,006 \\
\hline & $\mathrm{N}$ & 44 & 44 & 44 & 44 & 44 & 44 & 44 \\
\hline \multirow{3}{*}{$\begin{array}{l}\text { Occupational } \\
\text { Function } \\
\text { (Total) } 4\end{array}$} & CorrelationCoefficient & 0,235 & 0,003 & 0,003 & 0,201 &, $510(* *)$ &, $379\left(^{*}\right)$ &, $317(*)$ \\
\hline & Sig. (2-tailed) & 0,125 & 0,984 & 0,984 & 0,191 & 0,000 & 0,011 & 0,036 \\
\hline & $\mathrm{N}$ & 44 & 44 & 44 & 44 & 44 & 44 & 44 \\
\hline \multirow{3}{*}{$\begin{array}{l}\text { Emotional } \\
\text { Function } \\
\text { (Total) } 4\end{array}$} & CorrelationCoefficient & 0,080 & $-0,164$ & $-0,164$ & 0,104 &, $363(*)$ &, $479(* *)$ & ,323(*) \\
\hline & Sig. (2-tailed) & 0,604 & 0,287 & 0,287 & 0,501 & 0,015 & 0,001 & 0,032 \\
\hline & $\mathrm{N}$ & 44 & 44 & 44 & 44 & 44 & 44 & 44 \\
\hline \multirow{3}{*}{$\begin{array}{l}\text { Cognitive } \\
\text { Function } \\
\text { (Total) } 4\end{array}$} & CorrelationCoefficient & 0,104 & 0,050 & 0,050 & 0,257 & 0,253 &, $432(* *)$ &, $301(*)$ \\
\hline & Sig. (2-tailed) & 0,501 & 0,747 & 0,747 & 0,092 & 0,097 & 0,003 & 0,047 \\
\hline & $\mathrm{N}$ & 44 & 44 & 44 & 44 & 44 & 44 & 44 \\
\hline \multirow{3}{*}{$\begin{array}{l}\text { Social } \\
\text { Function } \\
\text { (Total) } 4\end{array}$} & CorrelationCoefficient & 0,074 & $-0,106$ & $-0,106$ & 0,081 & ,380(*) &, $489(* *)$ &, $401(* *)$ \\
\hline & Sig. (2-tailed) & 0,634 & 0,495 & 0,495 & 0,600 & 0,011 & 0,001 & 0,007 \\
\hline & $\mathrm{N}$ & 44 & 44 & 44 & 44 & 44 & 44 & 44 \\
\hline \multirow{3}{*}{$\begin{array}{l}\text { Fatigue } \\
\text { (Total) } 4\end{array}$} & CorrelationCoefficient & 0,141 & $-0,101$ & $-0,101$ & 0,073 &, $486(* *)$ &, $464(* *)$ &, $424(* *)$ \\
\hline & Sig. (2-tailed) & 0,363 & 0,513 & 0,513 & 0,636 & 0,001 & 0,002 & 0,004 \\
\hline & $\mathrm{N}$ & 44 & 44 & 44 & 44 & 44 & 44 & 44 \\
\hline \multirow[t]{3}{*}{ Pain (Total) 4} & CorrelationCoefficient & 0,120 & 0,060 & 0,060 & 0,114 &, $396(* *)$ &, $304\left(^{*}\right)$ &, $462(* *)$ \\
\hline & Sig. (2-tailed) & 0,436 & 0,698 & 0,698 & 0,462 & 0,008 & 0,045 & 0,002 \\
\hline & $\mathrm{N}$ & 44 & 44 & 44 & 44 & 44 & 44 & 44 \\
\hline \multirow[t]{3}{*}{ Dyspnea 4} & CorrelationCoefficient & $-0,115$ &,$- 298(*)$ &,$- 298(*)$ & $-0,019$ & 0,269 &, $406(* *)$ &, $305(*)$ \\
\hline & Sig. (2-tailed) & 0,459 & 0,049 & 0,049 & 0,905 & 0,078 & 0,006 & 0,044 \\
\hline & $\mathrm{N}$ & 44 & 44 & 44 & 44 & 44 & 44 & 44 \\
\hline \multirow[t]{3}{*}{ Insomnia 4} & CorrelationCoefficient & $-0,077$ & $-0,254$ & $-0,254$ & 0,010 &, $367\left(^{*}\right)$ &, $390(* *)$ & 0,200 \\
\hline & Sig. (2-tailed) & 0,620 & 0,096 & 0,096 & 0,948 & 0,014 & 0,009 & 0,193 \\
\hline & $\mathrm{N}$ & 44 & 44 & 44 & 44 & 44 & 44 & 44 \\
\hline \multirow{3}{*}{$\begin{array}{l}\text { Loss } \\
\text { Appetite } 4\end{array}$} & CorrelationCoefficient & 0,223 & 0,054 & 0,054 & 0,186 &, $566(* *)$ &, $612(* *)$ &, $463(* *)$ \\
\hline & Sig. (2-tailed) & 0,146 & 0,725 & 0,725 & 0,226 & 0,000 & 0,000 & 0,002 \\
\hline & $\mathrm{N}$ & 44 & 44 & 44 & 44 & 44 & 44 & 44 \\
\hline \multirow{3}{*}{$\begin{array}{l}\text { Constipation } \\
4\end{array}$} & CorrelationCoefficient & $-0,028$ & 0,207 & 0,207 & 0,101 & 0,275 &, $356\left(^{*}\right)$ &, $336\left(^{*}\right)$ \\
\hline & Sig. (2-tailed) & 0,855 & 0,178 & 0,178 & 0,512 & 0,070 & 0,018 & 0,026 \\
\hline & $\mathrm{N}$ & 44 & 44 & 44 & 44 & 44 & 44 & 44 \\
\hline \multirow[t]{3}{*}{ Diarrhea 4} & CorrelationCoefficient & 0,142 & $-0,137$ & $-0,137$ & 0,269 &, $555(* *)$ & 0,173 & 0,087 \\
\hline & Sig. (2-tailed) & 0,356 & 0,376 & 0,376 & 0,078 & 0,000 & 0,262 & 0,575 \\
\hline & $\mathrm{N}$ & 44 & 44 & 44 & 44 & 44 & 44 & 44 \\
\hline \multirow{3}{*}{$\begin{array}{l}\text { Financial } \\
\text { distress4 }\end{array}$} & CorrelationCoefficient & 0,136 & $-0,074$ & $-0,074$ & $-0,024$ & 0,281 &, $322(*)$ & 0,158 \\
\hline & Sig. (2-tailed) & 0,378 & 0,635 & 0,635 & 0,877 & 0,064 & 0,033 & 0,306 \\
\hline & $\mathrm{N}$ & 44 & 44 & 44 & 44 & 44 & 44 & 44 \\
\hline \multirow{3}{*}{$\begin{array}{l}\text { Global health } \\
\text { status } \\
\text { evaluation } \\
\text { (Total) } 4\end{array}$} & CorrelationCoefficient & $-0,011$ & 0,092 & 0,092 & $-0,039$ &,$- 441(* *)$ &,$- 336\left(^{*}\right)$ & $-0,270$ \\
\hline & Sig. (2-tailed) & 0,942 & 0,553 & 0,553 & 0,803 & 0,003 & 0,026 & 0,077 \\
\hline & $\mathrm{N}$ & 44 & 44 & 44 & 44 & 44 & 44 & 44 \\
\hline
\end{tabular}

${ }^{*} p<0.05,{ }^{* *} p<0.01$ 
Table 5. LC13 and toxicity correlation in the $4^{\text {th }}$ cycle.

\begin{tabular}{|c|c|c|c|c|c|c|c|c|}
\hline & & Anemia & $\begin{array}{l}\text { Leucopeni } \\
\text { a }\end{array}$ & Neutropenia & $\begin{array}{l}\text { Thrombocyt } \\
\text { openia }\end{array}$ & $\begin{array}{l}\text { Weight } \\
\text { loss }\end{array}$ & Nausea & Vomiting \\
\hline \multirow[t]{3}{*}{$\begin{array}{l}\text { Coughin } \\
\text { g } 4\end{array}$} & $\begin{array}{l}\text { CorrelationCoef } \\
\text { ficient }\end{array}$ & $-0,124$ & 0,000 & 0,000 & 0,043 & 0,165 &, $413(* *)$ & ,312(*) \\
\hline & Sig. (2-tailed) & 0,423 & 1,000 & 1,000 & 0,780 & 0,285 & 0,005 & 0,039 \\
\hline & $\mathrm{N}$ & 44 & 44 & 44 & 44 & 44 & 44 & 44 \\
\hline \multirow[t]{3}{*}{$\begin{array}{l}\text { Haemopt } \\
\text { ysis } 4\end{array}$} & $\begin{array}{l}\text { CorrelationCoef } \\
\text { ficient }\end{array}$ & $-0,058$ & 0,111 & 0,111 & 0,065 &, $311(*)$ &, $370(*)$ &, $386(* *)$ \\
\hline & Sig. (2-tailed) & 0,708 & 0,473 & 0,473 & 0,677 & 0,040 & 0,014 & 0,010 \\
\hline & $\mathrm{N}$ & 44 & 44 & 44 & 44 & 44 & 44 & 44 \\
\hline \multirow[t]{3}{*}{$\begin{array}{l}\text { Dyspnea } \\
\text { (ort) } 4\end{array}$} & $\begin{array}{l}\text { CorrelationCoef } \\
\text { ficient }\end{array}$ & $-0,174$ & $-0,209$ & $-0,209$ & 0,076 &, $341\left(^{*}\right)$ & 0,207 & 0,079 \\
\hline & Sig. (2-tailed) & 0,259 & 0,172 & 0,172 & 0,623 & 0,023 & 0,177 & 0,612 \\
\hline & $\mathrm{N}$ & 44 & 44 & 44 & 44 & 44 & 44 & 44 \\
\hline \multirow[t]{3}{*}{$\begin{array}{l}\text { Sore } \\
\text { mouth } 4\end{array}$} & $\begin{array}{l}\text { CorrelationCoef } \\
\text { ficient }\end{array}$ & 0,166 & 0,028 & 0,028 &, $379\left(^{*}\right)$ &, $422(* *)$ &, $358(*)$ & 0,172 \\
\hline & Sig. (2-tailed) & 0,282 & 0,859 & 0,859 & 0,011 & 0,004 & 0,017 & 0,266 \\
\hline & $\mathrm{N}$ & 44 & 44 & 44 & 44 & 44 & 44 & 44 \\
\hline \multirow[t]{3}{*}{$\begin{array}{l}\text { Dysphagi } \\
\text { a4 }\end{array}$} & $\begin{array}{l}\text { CorrelationCoef } \\
\text { ficient }\end{array}$ & 0,225 & 0,068 & 0,068 & 0,242 &, $519(* *)$ &, $364(*)$ &, $411(* *)$ \\
\hline & Sig. (2-tailed) & 0,142 & 0,663 & 0,663 & 0,114 & 0,000 & 0,015 & 0,006 \\
\hline & $\mathrm{N}$ & 44 & 44 & 44 & 44 & 44 & 44 & 44 \\
\hline \multirow[t]{3}{*}{$\begin{array}{l}\text { Neuropat } \\
\text { hy } 4\end{array}$} & $\begin{array}{l}\text { CorrelationCoef } \\
\text { ficient }\end{array}$ & 0,150 & $-0,020$ & $-0,020$ & 0,118 & 0,214 & 0,089 & 0,177 \\
\hline & Sig. (2-tailed) & 0,331 & 0,896 & 0,896 & 0,446 & 0,162 & 0,564 & 0,250 \\
\hline & $\mathrm{N}$ & 44 & 44 & 44 & 44 & 44 & 44 & 44 \\
\hline \multirow[t]{3}{*}{$\begin{array}{l}\text { Alopecia } \\
4\end{array}$} & $\begin{array}{l}\text { CorrelationCoef } \\
\text { ficient }\end{array}$ & $-0,027$ &,$- 327(*)$ &,$- 327(*)$ & $-0,211$ & 0,188 & $-0,024$ & $-0,149$ \\
\hline & Sig. (2-tailed) & 0,860 & 0,030 & 0,030 & 0,170 & 0,221 & 0,877 & 0,334 \\
\hline & $\mathrm{N}$ & 44 & 44 & 44 & 44 & 44 & 44 & 44 \\
\hline \multirow[t]{3}{*}{$\begin{array}{l}\text { Chest } \\
\text { pain } 4\end{array}$} & $\begin{array}{l}\text { CorrelationCoef } \\
\text { ficient }\end{array}$ & 0,184 & 0,231 & 0,231 & 0,152 & 0,247 & 0,223 &, $342(*)$ \\
\hline & Sig. (2-tailed) & 0,232 & 0,132 & 0,132 & 0,325 & 0,106 & 0,145 & 0,023 \\
\hline & $\mathrm{N}$ & 44 & 44 & 44 & 44 & 44 & 44 & 44 \\
\hline \multirow{3}{*}{$\begin{array}{l}\text { Pain in } \\
\text { arms and } \\
\text { shoulders } \\
4\end{array}$} & $\begin{array}{l}\text { CorrelationCoef } \\
\text { ficient }\end{array}$ & 0,066 & $-0,069$ & $-0,069$ & $-0,053$ &, $377(*)$ & 0,025 & 0,145 \\
\hline & Sig. (2-tailed) & 0,673 & 0,656 & 0,656 & 0,733 & 0,012 & 0,871 & 0,348 \\
\hline & $\mathrm{N}$ & 44 & 44 & 44 & 44 & 44 & 44 & 44 \\
\hline
\end{tabular}

${ }^{*} p<0.05,{ }^{* *} p<0.01$

vomiting; and between loss of appetite and nausea, vomiting and weight loss. Global health status evaluation was strongly correlated with weight loss, nausea and vomiting in a negative direction (Table 2).
In the evaluation of the relation of LC13 with toxicity in cycle 2, strong positive correlations were found between coughing and weight loss and nausea; between haemoptysis and weight loss, nausea and 
vomiting; between dyspnea and weight loss, nausea and vomiting. Similarly, strong positive correlations were observed between dysphagia and nausea/ vomiting and between neuropathy and weight loss (Table 3).

The relation between the QLQ-C30 and toxicity in the $4^{\text {th }}$ cycle showed that deterioration in physical function scale was strongly positively correlated with weight loss, nausea and vomiting; deterioration in occupational functions with weight loss; deterioration in emotional functions with nausea; deterioration in cognitive functions with nausea; and deterioration in social functions with nausea and vomiting. Also, fatigue was strongly positively correlated with weight loss, nausea-vomiting; pain scale with weight loss/vomiting; dyspnea with nausea and vomiting; insomnia with nausea; and loss of appetite with weight loss, nausea and vomiting. Global health status evaluation was strongly correlated with weight loss and weakly correlated with nausea in a negative direction (Table 4).

As a result of the examinations on LC13 and toxicity relation in cycle 4, strong positive correlations were determined between sore mouth and dysphagia; nausea and coughing; and coughing and dysphagia scales (Table 5).

\section{DISCUSSION}

Quality of life in lung cancer is how a patient's physical, emotional and social well-being is affected by the disease and the treatment process depending on the state of the disease and its treatment ${ }^{(15,16)}$. Besides the effectiveness of standard cancer treatment, response rates and survival time; QoL has become a significant criterion to determine how the patient's physical, emotional and social functions are affected by the disease itself and to plan the following treatment ${ }^{(4,5)}$. No significant difference was found in the comparisons of QoL parameters in patients over and under 60 years of age in this study. QoL is adversely affected by age in studies on healthy individuals whereas those conducted on cancer patients report that elderly individuals accept cancer more easily than the young, which make the former better than the latter in emotional and social aspects ${ }^{(6,17)}$.

Men had better physical and social functions in $1^{\text {st }}-2-$ nd $\mathrm{CT}$, and that men's social functions and global health status evaluation were better than women in $1^{\text {st }} \mathrm{CT}$. Similar with general cancer patients, women are reported to experience cancer more intensely and have higher anxiety ${ }^{(6,17,18)}$.

Education and income are factors affect QoL positively ${ }^{(19,20)}$. In this study, patients who completed primary or further education were observed to have better social functions in $1^{\text {st }} \mathrm{CT}$, better physical functions in $3^{\text {rd }}$ CT and occupational functions in $3^{\text {rd, }} 4^{\text {th }}$ $\mathrm{CT}$. Income statue did not correlate with QoL. Global health status of the patients without comorbidities was better than comorbid patients in first CT. Comorbidities increased with age. In NSCLC, although age is not an independent factor affecting survival, presence of comorbidities was correlated with increased mortality. In an observational study with 20.000 patients aged $>65$ years, age was considered more than comorbid diseases and treatment response rates were lower in elderly patients with less comorbidity in comparison to younger with more comorbid diseases ${ }^{(21)}$.

EORTC-C30, during the follow-up period, reveal an overall deterioration in functional scales, correlated with physical, emotional and cognitive functions. Symptom scale showed deterioration in fatigue, nausea-vomiting and insomnia scales. In stage III-IV NSCLC, improvement was found in emotional function on the functional scale and in insomnia, constipation and pain on the symptom scale ${ }^{(22)}$. Decrease was reported in the QLQ-C30 functional areas and the global health status as well as increases in alopecia, constipation and sore mouth ${ }^{(23)}$. In the evaluation of the QLQ-C30 during four cycles of CT, social functions deteriorated while constipation and financial distress increased with CT. As LC13 over four cycles was studied, alopecia and neuropathy increased together with $\mathrm{CT}$. 
Small cell lung cancer is a progressive tumor that responds to $\mathrm{CT}$ more rapidly and at higher rates. Improvements were observed in global health status, emotional and role functions with $\mathrm{CT}$ in patients with SCLC whereas only global health status improved in NSCLC (24). Similarly, when NSCLC was compared to SCLC during four cycles of $\mathrm{CT}$, we found occupational function to be better in the first cycle in NSCLC. Physical/occupational functions and global health status were better as well as symptoms of fatigue, pain and appetite in $3^{\text {rd }}-4^{\text {th }}$ cycles in SCLC. LC13 revealed that neuropathy was experienced more severely in $3^{\text {rd }}-4^{\text {th }}$ cycles, alopecia in $1^{\text {st }}$ cycle and pain in arms/shoulders in $3^{\text {rd }}$ cycle in NSCLC.

The physical/cognitive/occupational functions in $2^{\text {nd }}$ $4^{\text {th }}$ cycles; emotional functions in $3^{\text {rd }}$; financial status and social functions in $1^{\text {stand }}$ global status evaluation in $2^{\text {nd }}-4^{\text {th }}$ cycles were better in the complete/ partial response group. Less fatigue and dyspnea were experienced and appetite was better in $3^{\text {rd }}-4^{\text {th }}$; patients had less pain in cycle 4; neuropathy was less frequent in cycles $2^{\text {nd }}-4^{\text {th }}$; and less pain was felt in arms and shoulders in cycle 3 in the complete/partial response group.

Local progression or progression with distant metastases of the disease was likely to affect QoL with the newly occurring symptoms and findings. The fact that pain becomes more severe and restricting, performance scores fall, such symptoms as asthenia and weight loss increase due to the new symptoms may have an impact on the patient's physical and emotional status which in turn could limit social activities. These symptoms may increase anxiety and depression in patients. The most common and significant toxicities are hematologic and gastrointestinal ${ }^{(25,26)}$. In this study, the most frequent toxicity types were same. Anemia and neutropenia as well as fever, nausea-vomiting and cachexia were generally found to be at grade 1-2. Life threatening or mortal, grade $4^{\text {th }}-5^{\text {th }}$ level toxicities occurred very rarely. As the level of toxicity increased, QoL parameters deteriorated in a reverse correlation.
Lung cancer symptom scale revealed significant correlations between fatigue, haemoptysis, daily activities and QoL in first follow-up, dyspnea in $2^{\text {nd }}$, and between fatigue and hemoglobin/hematocrit values in $3^{\text {rd }}$. Nausea and/or vomiting may develop in $70-80 \%$ receiving CT and affect the patient's diet, performance and QoL ${ }^{(26,27,28)}$. We similarly observed nausea and vomiting to deteriorate QoL significantly. Being aware of the adverse effects of CT is just as important as the effectiveness of the treatment. During patient check-ups, the primary concern is following the disease and assessing treatment response. It is known that questioning the treatment induced adverse effects and QoL is disregarded ${ }^{(29)}$.

As one can assume, presence of comorbidities turned out to be one of the most critical factors affecting both the decision and method for treatment and QOL parameters. Patients were observed to have deteriorated social functions, increased constipation, alopecia and neuropathy and deteriorated economic parameters together with CT. Since SCLC is a tumor that responds rapidly/well to CT, QoL were observed to improve better in these patients in comparison to NSCLC. QoL of patients with complete/ partial response to CT were affected positively when compared to patients accepted as stable/progressive. A significant relation was found between toxicity and deterioration in QoL.

As a limitation the study lacks of an analysis with control group

\section{CONCLUSION}

While planning the treatment, clinicians should provide treatment and care with a multidisciplinary teamwork understanding and "holistic treatment and care" approach in order to improve the patients' QoL. Social and psychological support should be planned besides to medical treatment.

In the treatment of advanced stage lung cancer today, it is still among the main purposes to improve QoL or not to disturb it further as well as extending 
lifetime. In patients receiving chemotherapy, accompanying comorbidities, low educational level, socioeconomic status and treatment oriented hematologic and gastrointestinal toxicities are the most significant parameters affecting QoL.

Ethics Committee Approval: S.B.U. Dr. Suat Seren Chest Diseases and Surgery Training and Research Hospital Ethics Committee approval was obtained (25.07.2014/7699).

\section{Conflict of Interest: There is no conflict of interest. Funding: None.} Informed Consent: Was obtained.

\section{REFERENCES}

1. Yang SC, Lai WW, Hsiue TR, et al. Health-related quality of life after first-line anti-cancer treatments for advanced nonsmall cell lung cancer in clinical practice. Qual Life Res. 2016;25:1441-9. [CrossRef]

2. Lee LJ, Chung CW, Chang YY, et al. Comparison of the quality of life between patients with non-small-cell lung cancer and healthy controls. Qual Life Res. 2011;20:415-23. [CrossRef]

3. Mannion E, Gilmartin JJ, Donnellan P, Keane M, Waldron D. Effect of chemotherapy on quality of life in patients with non-small cell lung cancer. Support Care Cancer. 2014;22:1417-28. [CrossRef]

4. Gultekin Z, Pinar G, Pinar T, et al. Health-related quality of life and health care services expectations of the patients with lung cancer. Int J Hematol Oncol. 2008;18:99-106.

5. Lozano R, Naghavi M, Foreman K, et al. Global and regional mortality from 235 causes of death for 20 age groups in 1990 and 2010: a systematic analysis for the Global Burden of Disease Study 2010. Lancet. 2012;380:2095-128. [CrossRef]

6. Pandey M. Sarita G. Devi N. Distress, anxiety, and depression in cancer patients undergoing chemotherapy. World J Surgical Oncology. 2006;4:68-74. [CrossRef]

7. Dai YL, Yang CT, Chen KH, Tang ST. Changes in and determinants of quality of life in patients with advanced non-smallcell lung cancer undergoing initial chemotherapy. J Nurs Res. 2017;25(3):203-15. [CrossRef]

8. Aaronson NK, Ahmedzai S, Bergman B, et al. The European Organization for Research and Treatment of Cancer QLQC30: a quality-of-life instrument for use in international clinical trials in oncology. J Natl Cancer Inst. 1993;85:365-76. [CrossRef]

9. Oken MM, Creec RH, Tormey DC, et al. Toxicity and response criteria of the Eastern Cooperative Oncology Group. Am J Clin Oncol. 1982;5:649-55. [CrossRef]

10. Yates JW, Chalmer B, McKegney FP. Evaluation of patients with advanced cancer using the Karnofsky performance status. Cancer. 1980;45:2220-4. [CrossRef]

11. Common Terminology Criteria for Adverse Events (CTCAE). US Departmant of Health and Human Services, National Cancer Institute. Version 4.03, Published: June 2010.

12. Guzelant A, Goksel T, Ozkok S. The European Organization for Research and Treatment of Cancer QLQ-30: an examination into the cultural validity and reliability of Turkish version of the EORTC QLQ-30. Eur J Cancer Care. 2004;13:135-44. [CrossRef]

13. Vallières E, Shepherd FA, Crowley J, et al. The IASLC Lung Cancer Staging Project: proposals regarding the relevance of TNM in the pathologic staging of small cell lung cancer in the forthcoming (seventh) edition of the TNM classification for lung cancer. J Thorac Oncol. 2009;4:1049-59. [CrossRef]

14. Bergman B, Aaronson NK, Ahmedzai S, Kaasa S, Sullivan M. The EORTC QLQ-LC13: A moduler supplement to the EORTC core quality of life questionnaire (QLQ-C30) for use in lung cancer clinical trials. EORTC Study Group on Quality of Life. Eur J Cancer. 1994;30A:635-42. [CrossRef]

15. Lazovich D, Robien K, Cutler G, Virnig B, Sweeney C. Quality of life in a prospective cohort of elderly women with and without cancer. Cancer. 2009;115(18 Suppl):4283-97. [CrossRef]

16. Myrdal G, Valtysdotir S, Lambe M, Stahle E. Quality of life following lung cancer surgery. Thorax. 2003;58:194-7. [CrossRef]

17. Alacacioglu A, Yavuzsen T, Dirioz M. Changes in anxiety levels patients with cancer receiving chemotherapy. Int J Hematol Oncol. 2007;17:17-9.

18. Nursen $O$, Nahcivan A. Turkish language equivalence of the exercise of self-care agency scale. Western J Nursing Res. 2004;7:813-24. [CrossRef]

19. Loscocco KA, Spitze G. Working conditions, social support, and the well-being of female and male factory workers. J Health Soc Behav. 1990;31:313-27. [CrossRef]

20. Evans C, Trudeau E, Mertzanis P. Development and validation of the pain treatment satisfaction scale (ptss): A patient satisfaction questionnaire for use in patients with chronic or acute pain. Pain. 2004;112:254-66. [CrossRef]

21. Wang S, Wong ML, Hamilton N, Davoren JB, Jahan TM, Walter LC. Impact of age and comorbidity on non-small-cell lung cancer treatment in older veterans. J Clin Oncol. 2012;30:1447-55. [CrossRef]

22. Gupta D, Braun DP, Staren ED. Association between changes in quality of life scores and survival in non-small cell lung cancer patients. Eur J Cancer Care. 2012;21:614-22. [CrossRef]

23. Montazeri A, Hole DJ, Milroy R, McEwen J, Gillis CR. Quality of life in lung cancer patients: does socioeconomic status matter? Health Qual Life Outcomes. 2003;9:1:19. [CrossRef]

24. Bircan A, Berktas BM, Bayiz H. Effects of chemotherapy on quality of life patients with lung cancer. Turkish Respir J. 2003;4:61-6.

25. Goktalay T, Akin M, Aydemir Ö. The assessment of quality of life of lung cancer patients. Solunum Hast Derg. 2002;13:26570.

26. Eser S, Goksel T, Erbaycu AE, et al. Comparison of generic and lung cancer-specific quality of life instruments for predictive ability of survival in patients with advanced lung cancer. SpringerPlus. 2016;5:1833. [CrossRef]

27. Abuzallouf S, Wright J, El Hattab O. Anemia in patients with advanced or metastatic non-small cell lung cancer. Gulf J Oncol. 2007;1:17-21.

28. Schwartzberg LS. Chemotherapy - induced nausea and vomiting: clinician and patient perspectives. J Support Oncol. 2007;5(suppl 1):5-12.

29. Atasoy BM, Unsal DK, Dincbas FO, Yamaç D, Okkan S, İçli F. Yan etki değerlendirilmesi ve kaydı üzerine anket sonuçları: Türk onkoloji grubu (TOG) erken-geç yan etkiler çalışma grubu araştırması. Turk Oncol J. 2007;22:13-9. 